\title{
REFLET DE LA PANDÉMIE DE COVID-19 DANS LES DICTIONNAIRES DE LA LANGUE FRANÇAISE
}

doi.org/10.15452/SR.2021.21.0008

ORCID ID: 0000-0003-3529-9578

\author{
Dagmar Koláříková \\ Université de Bohême de l'Ouest de Plzeň \\ République tchèque \\ dakola@seznam.cz
}

Résumé. Les langues sont bien adaptées pour refléter les changements qui surviennent dans la vie de leurs utilisateurs. La pandémie de Covid-19, par sa spécificité, est généreuse envers la langue française qui a vite créé et emprunté des unités lexicales simples et complexes, un nouveau vocabulaire spécialisé qui reflète les transformations survenues dans la société. Des termes médicaux comme coronavirus (type de virus) et COVID-19 (maladie causée par le SARS-CoV-2) font désormais partie du vocabulaire quotidien. De nouveaux mots et de nouveaux sens font généralement leur entrée dans les dictionnaires une fois que les éditeurs ont suffisamment de preuves pour démontrer leur installation dans l'usage réel. Certaines lexies mettent donc des décennies à faire leur apparition dans les dictionnaires. Sur la base de ce qui précède, la question se pose : comment la pandémie a-t-elle influencé les éditeurs de dictionnaires ? La présente étude tente d'étudier les nouveaux mots et expressions français qui ont émergé à la suite de la crise du Covid-19 et ont été inclus dans les dictionnaires de la langue française. Le corpus recueilli pour cette étude est constitué de néologismes (le concept de néologisme peut être trompeur ici car la théorie lexicographique caractérise les néologismes comme des mots qui n'ont pas été inclus dans les dictionnaires généraux), provenant de divers domaines, de nouveaux mots et expressions et de nouveaux sens qui ont fait leur entrée dans les dictionnaires papier Le Petit Robert 2022 et Le Petit Larousse illustré 2022 et les dictionnaires en ligne le Grand dictionnaire terminologique et le Wiktionnaire. Le Petit Robert a ajouté 26 nouveaux mots et sens dans son édition 2022 et 48 nouveaux mots et sens, du cluster au coronapiste (piste cyclable créée au début de la pandémie de COVID-19), sont entrés dans le dictionnaire Le Petit Larousse illustré 2022. Alors, la présente étude montre comment les dictionnaires français ont 
su s'adapter aux changements que la pandémie de Covid-19 a occasionnés. Pendant cette crise sanitaire, la langue française a montré un dynamisme extraordinaire.

Mots clés. COVID-19. Dictionnaire. Langue française. Néologisme. Nouveau mot. Nouveau sens.

Abstract. The Reflection of the COVID-19 Pandemic in Dictionaries of the French Language. Languages adapt to reflect changes taking place in the life of users. The COVID-19 pandemic, by its specificity, has had an enriching effect on the French language, which quickly created and borrowed simple and complex lexical units, a new specialized vocabulary reflecting the transformations that have occurred in the society. Medical terms like coronavirus (type of virus) and COVID-19 (disease caused by SARS-CoV-2) became a part of everyday conversation. New words and new meanings are usually added to dictionaries once editors have enough evidence to demonstrate continued historical use; therefore, they must be used over a significant period of time to earn their place in dictionaries. Based on the above, the question arises: how has the epidemic impacted dictionary editors? The present study attempts to investigate the new French words and expressions that emerged in the wake of the COVID-19 crisis and were added to dictionaries of the French language. The corpus collected within this study comprises neologisms (the concept of neologism can be misleading here, because the lexicography theory characterizes neologisms as words which have not been included in current dictionaries) from various fields, new words or expressions and new meanings which have been added to the Petit Robert and the Petit Larousse illustré and the online dictionaries Grand dictionnaire terminologique and Wiktionnaire. The French dictionary Le Petit Robert has added 26 new words and meanings to its 2022 dictionary, and 48 new words and meanings, from cluster to coronapiste (a cycle lane introduced during the COVID-19 crisis), have entered the French dictionary Le Petit Larousse 2022. The present study shows how French dictionaries have been able to adapt to the changes brought about by the COVID-19 pandemic. During this health crisis, the French language has been extraordinarily dynamic.

Keywords. COVID-19. Dictionary. French language. Neologism. New meaning. New word. 


\section{Introduction}

Toute langue vivante est en constante évolution. Au fil du temps, de nouveaux mots naissent, des mots obsolètes disparaissent, certains mots se modifient ou changent de sens. Ainsi, les langues s'adaptent spontanément aux mutations de la société et aux nouvelles exigences expressives des parlants.

Ce sont surtout les événements majeurs qui ont un grand impact sur les langues et en particulier sur leur vocabulaire. Dans la catégorie « événements majeurs », la pandémie de Covid-19 qui chamboule nos vies depuis la fin de 2019 se classe très haut dans le palmarès de l'histoire moderne. Les années 2020 et 2021 voient alors proliférer des termes comme coronavirus et Covid-19 qui, étant rapidement entérinés par les utilisateurs, deviennent des vedettes instantanées du lexique à cette époque. Le premier, désignant une catégorie de virus, n'est pas une création millésimée 2020 (le mot est âgé plus d'un demi-siècle car les coronavirus ont été identifiés dans les années 1960) mais, au cours des deux dernières années, ce terme spécialisé se diffuse massivement dans les médias et dans la langue courante. Pour ce qui est le terme Covid-19 désignant la maladie causée par un coronavirus apparu à la fin de 2019, celui-ci date bien de 2020. Il en résulte que certains néologismes liés à la pandémie sont des inventions complètes, d'autres sont formés à partir de termes existants, cela veut dire que ceux-ci ont trouvé un nouveau sens à travers cette pandémie.

Mais les deux termes susmentionnés sont-ils vraiment des néologismes ? Le mot est généralement considéré comme nouveau dès l'instant de sa naissance à son intégration dans un dictionnaire général, ce que souligne par exemple Mortureux qui définit le néologisme comme « un mot reconnu à la fois, comme nouveau et susceptible de se lexicaliser. Car on l'applique essentiellement à des mots en cours de diffusion, avant que leur diffusion n'ait abouti à les faire enregistrer dans les dictionnaires généraux » (Mortureux, 1997 : 105).

Pour pouvoir répondre à cette question, il faut tout d'abord trouver les réponses aux questions suivantes : dans ce contexte évolutif, les principaux éditeurs de dictionnaires (Larousse et Le Robert), mettent-ils à jour leurs ouvrages pour que les termes liés à la pandémie de Covid-19 et utilisés largement par la population y trouvent leur définition? Les mêmes mots font-ils leur entrée dans les millésimes 2021 et 2022 du Petit Robert et du Petit Larousse illustré ? Ce sont des questions qui nous semblent intéressantes, surtout que les linguistes constatent généralement un retard d'enregistrement des mots nouveaux dans la nomenclature des dictionnaires, ce que nous évoquerons dans la partie théorique.

L'objectif de cette étude est donc de répertorier les nouveaux termes marqués par la crise sanitaire qui ont fait leur apparition dans les pages du Petit Robert et du Petit Larousse illustré pour les millésimes 2021 et 2022. Pour ce faire, nous analyserons le corpus DiCo (Dictionnaires Comparés) publié par le Club d'orthographe de Grenoble et les présentations des mots nouveaux du Petit Robert et du Petit Larousse illustré et nous essaierons de comparer les résultats obtenus avec ceux provenant de l'analyse effectuée dans deux dictionnaires en ligne (notamment, le Grand dictionnaire terminologique et le Wiktionnaire) pour voir si ces derniers sont plus réactifs à la naissance des néologismes en rapport avec la pandémie que les dictionnaires papier. L'exigence méthodologique d'obtenir des réponses plus variées, provenant d'une part d'un 
dictionnaire plutôt «officiel» (en plus, prenant en compte le contexte référentiel québécois) et d'autre part d'un dictionnaire universel rédigé par des contributeurs bénévoles, a conditionné notre choix des dictionnaires en ligne.

\section{2. À propos de la dictionnarisation d'une forme ou d'un sens nouveaux}

Comme nous l'évoquons dans l'introduction et que l'indique aussi Cusin-Berche (1999), la non-intégration dans la nomenclature d'ouvrages lexicographiques est utilisée par un grand nombre d'auteurs, par exemple Cabré (1998), Guilbert (1975), Rey-Debove (1971) et d'autres, comme critère néologique. Néanmoins, d'après Sablayrolles :

L'absence d'une lexie dans un dictionnaire ne signifie en effet pas pour autant qu'elle soit néologique. Des contraintes matérielles (de place, de mise en page, en particulier) peuvent conduire à négliger des lexies peu fréquentes. Des mots ou tournures sentis comme archaïques ou désuets peuvent aussi être délibérément omis pour traiter plus à fond le vocabulaire courant (Sablayrolles, $2000: 180)$.

En plus, il est notoire que les éditeurs de dictionnaires généraux de langue restent prudents d'enregistrer les mots nouveaux. Ces derniers n'entrent généralement pas dans la nomenclature des dictionnaires que lorsque leur diffusion et réception sociales ont atteint un seuil suffisant pour que leur lexicalisation soit considérée comme accomplie. Le critère essentiel d'entrée d'un mot dans un dictionnaire est donc son installation dans l'usage réel. Sablayrolles (2019: 63) explique l'entrée tardive des néologismes dans les dictionnaires comme il suit : « Il faut se méfier de la doxa qui veut qu'un mot soit néologique parce qu'il n'est pas dans le dictionnaire. C'est l'inverse qui est vrai : un mot entre dans un dictionnaire quand et parce qu'il n'est plus un néologisme ».

De toute évidence, c'est le facteur temps qui peut aussi être vu comme garant de la stabilité de l'emploi d'une lexie. Pourtant, la durée entre la naissance de celle-ci et son accueil par les dictionnaires peut varier considérablement. Sablayrolles $(2000: 202)$ souligne que «plusieurs siècles sont parfois nécessaires pour qu'une lexie éclose complètement ». Le même auteur, s'appuyant sur les travaux de Elchacar et Martinez (2008), donne un exemple concret dans l'article intitulé Alain Rey, le linguiste et les mots nouveaux (2011) où il montre que certaines lexies mettent des décennies à faire leur apparition dans le Petit Robert, avec une moyenne d'une quarantaine/cinquantaine d'années pour les éditions de 1998 à 2009.

Néanmoins, il est à noter qu'il n'y a pas de format temporel préétabli. Dans les dictionnaires, il est possible de relever plusieurs mots enregistrés deux ou trois ans après leur première attestation, ce que démontre par exemple Bouzidi (2010) en mentionnant entre autres le mot airbag dont la durée entre la première attestation et sa dictionnarisation n'est que deux ans (il est daté de 1992 dans le Petit Robert et inséré par le Petit Larousse illustré en 1994). En effet, cette durée entre la naissance et l'accueil par un dictionnaire peut être encore plus courte si l'on prend en compte le fait que souligne Sablayrolles (2011) « que le millésime est trompeur, car le 
dictionnaire paraît six mois avant le début de l'année civile dont il porte le millésime et qu'il faut donc diminuer d'un an la date indiquée ». Ainsi, selon l'auteur, des mots peuvent entrer dans les dictionnaires l'année même de leur première attestation ou un an après. Une nouvelle expression peut alors être dictionnarisée assez rapidement, l'important est qu'elle soit largement utilisée par les médias et le grand public.

Il en résulte qu'il est impossible de répondre à la question : jusqu'à quand une lexie est-elle néologique ? Comme le dit Sablayrolles (2019), il est difficile de donner une réponse valable pour tous les cas car la durée de la néologicité est tout sauf uniforme et varie en fonction de plusieurs paramètres, en particulier de nature sociolinguistique.

Chaque dictionnaire a sa propre politique éditoriale. Mais, dans la plupart des cas, les dictionnaires français essaient de faire entrer des mots poussés par l'actualité mais aussi dont on peut supposer qu'ils vont rester dans la langue française. Comme l'affirment Boulanger et al. (2003 : 157), « le dictionnaire demeure le lieu le plus pertinent pour suivre la circulation des mots dans la langue ». En accueillant des formes et des sens nouveaux chaque année, il nous renseigne sur l'évolution du vocabulaire qui reflète celle de la société.

Dès que la pandémie de Covid-19 est apparue, il y avait donc une nécessité à nommer les choses et en parler. Beaucoup de formes et de sens nouveaux apparaissent brusquement dans tous les médias ou sur les réseaux sociaux et passent rapidement dans la langue courante. Dans la partie suivante, nous essaierons alors de relever quels mots ou sens nouveaux ont été dictionnarisés au cours des deux dernières années (c'est-à-dire, pour les millésimes 2021 et 2022).

\section{Mots et sens nouveaux liés à la pandémie de Covid-19 ayant fait leur entrée aux dictionnaires de la langue française}

Avant de passer à l'analyse des mots liés à la crise sanitaire, qui sont entrés entre 2020 et 2021 dans les dictionnaires consultés, revenons encore aux termes mentionnés dans l'introduction - coronavirus et Covid-19. Comme il a déjà été expliqué, ils sont deux choses différentes. Le coronavirus (virus à couronne dont le nom scientifique est le Sars-Cov-2) est une famille du virus qui paralyse aujourd'hui le monde entier et le Covid-19 désigne la maladie provoquée par ce virus.

Mais, depuis le début de la pandémie, une confusion règne entre les deux termes, ce qui suscite de nombreux débats autour du genre du mot Covid-19 qui provient de l'acronyme corona virus disease (maladie à coronavirus, en français). L'Académie française et l'Office québécois de la langue française ont opté pour le féminin (la Covid) en s'appuyant sur l'explication que « les sigles et acronymes ont le genre du nom qui constitue le noyau du syntagme dont ils sont une abréviation »' (ici, il s'agit de disease se traduisant par maladie en français, un nom féminin), alors que l'usage du masculin est majoritaire dans l'Europe francophone. Même les dictionnaires Le Petit Robert et Le Petit Larousse illustré n'ont pas réussi à trancher le débat. Dans leurs éditions 2022, ils ne tombent pas d'accord sur la question du genre. Le Petit Robert indique qu'il

1 Académie française. Le covid 19 ou La covid 19. https://www.academie-francaise.fr/le-covid-19-ou-lacovid-19 [11-08-2021]. 
s'agit d'un nom masculin ou féminin en plaçant le genre masculin en premier, tandis que son concurrent le Petit Larousse illustré le qualifie de « féminin ou masculin » (donc le genre féminin en premier). Dans la présentation du dictionnaire 2022, Le Robert argumente que « c'est l'usage qui fait loi. Si le féminin est adopté au Canada francophone, le masculin est pour l'instant majoritaire en France, où l'avis de l'Académie française a été rendu tardivement, alors que le masculin était déjà bien implanté ». ${ }^{2}$ En revanche, le Petit Larousse illustré estime le féminin plus correct, tout comme l'Académie française, se référant à la maladie causée par le virus mais en même temps précise que l'utilisation de ce mot au masculin est courante car le masculin est influencé sans doute par le mot conoranvirus qui s'emploie au masculin. Covid est alors entré dans les dictionnaires sous les deux genres, on a le choix de dire « le covid » ou « la covid ». La recherche sur la plateforme Néoveille (effectuée le 30 août 2021), confirme que les tendances varient selon les pays. La figure 1 montre une large préférence pour le covid en France et la figure 2 pour la covid au Canada (pas de distinction minuscule-majuscule).

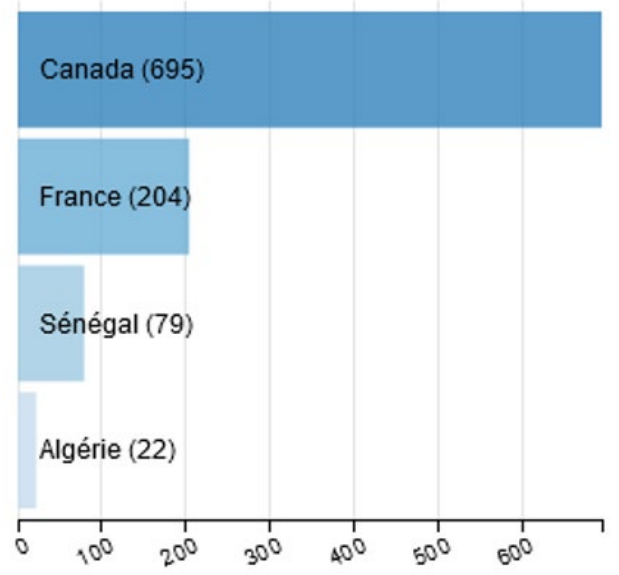

Fig.1 : Répartition par pays : le covid (Néoveille)

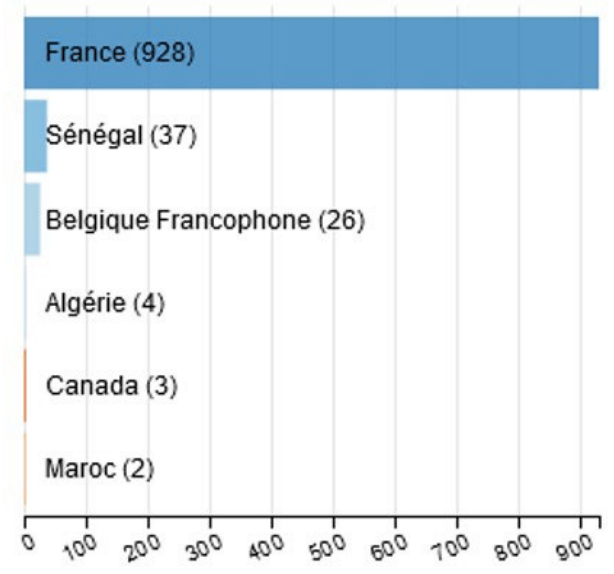

Fig. 2 : Répartition par pays : la covid (Néoveille)

Au-delà de la question du genre, c'est aussi l'orthographe du mot qui fait l'objet d'hésitations. On retrouve différentes graphies du mot : covid-19, Covid-19, COVID-19 ou covid 19 (sans trait d'union ni majuscule, ce qui est recommandé par l'Académie française). Si le Petit Robert comme le Petit Larousse illustré définissent le concept covid comme une « maladie infectieuse, très contagieuse, causée par un coronavirus », ${ }^{3}$ ils ne se sont en revanche pas non plus mis d'accord sur le choix entre la minuscule et la majuscule : pour le premier, il s'écrit avec la minuscule, sauf dans le cas de Covid-19 (le Petit Robert distingue ainsi le terme générique de covid, comme

2 Le Robert. Le Robert présente ses dictionnaires et mots nouveaux 2022. https://manuscritdepot.com/ dictionnaire_le_robert_mots_nouveaux_2022.pdf [12-08-2021].

3 Le Robert, dico en ligne. Covid. https://dictionnaire.lerobert.com/definition/covid [12-08-2021]. 
dans l'exemple suspicion de covid, et celui spécifique qui s'écrit avec la majuscule) tandis que le Petit Larousse illustré préconise la majuscule et donne comme graphies possibles COVID-19 ou Covid-19.

\section{1 Étude comparative des formes et sens nouveaux de la pandémie introduits dans le Petit Robert et le Petit Larousse illustré}

La crise sanitaire n'a pas seulement apporté de nouveaux termes pour désigner le virus, ses variants et la maladie mais aussi ceux qui sont liés à toutes les situations nouvelles résultant de la pandémie et aux conséquences sociétales de celle-ci. Pour voir si les dictionnaires sont ouverts à l'intégration de ce vocabulaire, nous avons effectué une étude comparative entre deux dictionnaires choisis - Le Petit Robert et Le Petit Larousse illustré.

Après l'analyse des mots nouveaux intégrés dans le dictionnaire Le Petit Robert 2021 (paru le 4 juin 2020), nous pouvons constater que le calendrier de production du dictionnaire papier n'a pas permis d'introduire les mots de la pandémie. Il en va de même pour le Petit Larousse illustré 2021 (publié le 3 juin 2020) qui n'intègre rien (sauf une ligne ajoutée à l'article coronavirus) au sujet de la pandémie. Mais les éditeurs ont réussi à intégrer quelques-uns de ces mots dans la version informatisée (à titre d'exemple, nous pouvons mentionner immunité collective, patient zéro, cluster, confinement, etc. qui ont été introduits dans la version en ligne du Petit Robert en 2020). Les versions informatisées prennent ainsi un peu d'avance sur celles en papier. Alors, les deux versions se désynchronisent, ce qui n'est pas habituel mais témoigne de la nécessité de réagir en temps réel.

Ce n'est donc que dans leurs éditions 2022 (sorties au mois de juin 2021) où le lexique lié à la crise sanitaire prédomine parmi les mots nouvellement intégrés dans les deux dictionnaires (26 nouveaux mots ou sens ont fait leur apparition dans le Petit Robert 2022 et 48 nouveaux mots et sens dans le Petit Larousse illustré 2022). Il est cependant intéressant à noter que l'analyse de ce vocabulaire nous montre que ce ne sont pas nécessairement les mêmes mots qui ont fait leur entrée dans les deux dictionnaires choisis, nous n'en avons relevé que seize. Pour l'essentiel, on y trouve ceux qui sont particuliers à chacun d'eux.

Néanmoins, nous commencerons par 16 termes qui ont fait leur entrée dans le millésime 2022 des deux dictionnaires. Pour la plupart, ces mots existaient déjà dans la langue française, mais, durant la crise sanitaire, ils ont pris un nouveau sens. Par exemple, l'anglicisme cluster faisait déjà partie du vocabulaire français et, en musique, il désignait la résonance de plusieurs notes jouées simultanément avec le poing, la paume ou l'avant-bras tandis que la crise sanitaire lui a donné un sens différent, celui d'un foyer épidémique. Il en va de même pour le confinement employé autrefois pour désigner l'interdiction à un malade de quitter la chambre, puis le fait de rassembler des volailles dans un espace délimité, notamment dans le contexte de l'épidémie de grippe aviaire. Alors que de nombreux pays ont adopté l'anglicisme lockdown pour indiquer la mesure de restriction consistant à enjoindre la population à demeurer autant que possible dans un lieu d'habitation déterminé afin de tenter de limiter la propagation du nouveau coronavirus, les francophones préfèrent un terme français et parlent de confinement. Et depuis le début de la crise sanitaire, de nombreux néologismes sont formés à partir de cette 
racine latine confins, correspondant à des étapes vécues par la société : déconfinement désignant la fin du confinement ou reconfinement pour indiquer un nouveau confinement.

Le tableau 1 donné ci-dessous présente tous les mots liés à la pandémie qui ont été intégrés dans les deux dictionnaires millésimés 2022, bénéficiant, dans la plupart des cas, d'un glissement de sens. Parmi eux, les mots qui n'existaient pas avant 2020 sont rares (Covid).

\begin{tabular}{|c|c|}
\hline $\begin{array}{l}\text { Mot entré dans les deux } \\
\text { dictionnaires }\end{array}$ & Définition \\
\hline Asymptomatique & $\begin{array}{l}\text { Ce dit d'un malade qui ne présente aucun symptôme clinique } \\
\text { (il qualifiait auparavant seulement une maladie). }\end{array}$ \\
\hline Cluster & Foyer épidémique. \\
\hline Confinement & Action de confiner (= forcer à rester dans un espace limité). \\
\hline Covid & $\begin{array}{l}\text { Maladie infectieuse et contagieuse causée par un corona- } \\
\text { virus. }\end{array}$ \\
\hline Déconfinement & Levée du confinement. \\
\hline Déconfiner & Mettre fin au confinement de (qqn). \\
\hline Distanciation sociale/physique & $\begin{array}{l}\text { Fait de maintenir une distance de sécurité entre les per- } \\
\text { sonnes pour des motifs sanitaires. }\end{array}$ \\
\hline Foyer & Lieu d'où se propage une maladie. \\
\hline Gestes/mesures barrières & $\begin{array}{l}\text { Précautions prises dans la vie quotidienne pour limiter la } \\
\text { propagation d'un virus, d'une maladie. }\end{array}$ \\
\hline Immunité collective & $\begin{array}{l}\text { Propriété permettant de casser la chaîne de transmission } \\
\text { d'une maladie au sein d'une population. }\end{array}$ \\
\hline Jauge & Capacité d'accueil d'un lieu public. \\
\hline Patient zéro & $\begin{array}{l}\text { Première personne ayant été contaminée lors d'une épidé- } \\
\text { mie. }\end{array}$ \\
\hline Rubalise & $\begin{array}{l}\text { Ruban en plastique de couleurs vives servant soit à délimiter } \\
\text { une zone particulière, parfois dangereuse, et en interdire l'ac- } \\
\text { cès, soit à baliser un parcours. }\end{array}$ \\
\hline Téléconsultation & $\begin{array}{l}\text { Consultation médicale à distance, grâce aux moyens de } \\
\text { télécommunication. }\end{array}$ \\
\hline Traçage (numérique) & $\begin{array}{l}\text { Suivi des déplacements de qqn. (à partir des données du } \\
\text { téléphone mobile en cas de traçage numérique). }\end{array}$ \\
\hline Virus aéroporté & $\begin{array}{l}\text { Virus transmis par des micro-organismes en suspension dans } \\
\text { l'air. }\end{array}$ \\
\hline
\end{tabular}

Tableau 1 : Les mêmes termes entrés dans le millésime 2022 du Petit Robert et du Petit Larousse illustré

Il ressort de cette liste que les deux dictionnaires ont surtout intégré les mots liés au domaine médical (par exemple, le patient zéro que l'on s'est efforcé de retrouver à l'origine de la formation de clusters, emprunt à l'anglais, entré en concurrence avec plusieurs syntagmes français comme foyer épidémique, foyer de contagion, foyer de contamination ou foyer tout court) mais aussi aux restrictions sur les gens (par exemple, les gestes barrières recommandés pour éviter la pro- 
pagation du virus, parmi lesquels figurent la distanciation sociale et/ou la distanciation physique, les deux sont des calques de l'anglais social distancing et physical distancing).

Néanmoins, d'autres termes qui ont fait leur apparition dans les pages du Petit Robert et du Petit Larousse illustré millésimés 2022 font également écho à la pandémie de Covid-19. Le tableau 2 contient tous les formes ou sens nouveaux que nous avons relevés par l'analyse des deux dictionnaires choisis et dont la présence nous avons aussi vérifié dans deux dictionnaires en ligne, notamment dans Le Grand dictionnaire terminologique (banque de fiches terminologiques rédigées par l'Office québécois de la langue française ou des partenaires de l'Office) et le Wiktionnaire (partie francophone du projet de dictionnaire multilingue de la fondation Wikimedia). ${ }^{4}$ Pour chacun des mots nous noterons la présence $(\mathrm{X}) /\left(\mathrm{X}^{\star 5}\right)$ ou l'absence $(---) /\left(--{ }^{\star 6}\right)$ d'une forme nouvelle ou d'un sens nouveau dans chaque dictionnaire composant notre corpus. Nous donnerons aussi une définition pour chacun des termes. Puis nous dégagerons un certain nombre de constats résultant de la comparaison des données obtenues par les dictionnaires consultés.

\begin{tabular}{|l|l|l|l|l|l|}
\hline $\begin{array}{l}\text { Mot entré dans un } \\
\text { des dictionnaires }\end{array}$ & PR & PLI & GDT & Wik. & Définition \\
\hline Aérosolisation, n. & $X$ & --- & --- & $X$ & $\begin{array}{l}\text { Diffusion aérienne de fines particules par } \\
\text { aérosol. }\end{array}$ \\
\hline $\begin{array}{l}\text { Antivaccin, adj., n. } \\
\text { Fam. antivax }\end{array}$ & X & X* & --- & $X$ & Qui est hostile à la vaccination. \\
\hline Cas contact & $X$ & ---7 & --- & $X$ & $\begin{array}{l}\text { Personne qui a été en contact avec une per- } \\
\text { sonne contaminée par le virus du Covid-19. }\end{array}$ \\
\hline $\begin{array}{l}\text { Click and collect } \\
\text { Recomm. off. } \\
\text { Cliqué-retiré }\end{array}$ & --- & $X$ & $X$ & $X$ & $\begin{array}{l}\text { Service permettant à un client de commander } \\
\text { un produit en ligne et de venir le retirer rapide- } \\
\text { ment sur le point de vente. }\end{array}$ \\
\hline $\begin{array}{l}\text { Confiné, adj., n. } \\
\text { Confiner }\end{array}$ & $---*$ & $X$ & --- & --- & $\begin{array}{l}\text { Se dit d'une personne soumise à un confine- } \\
\text { ment (sanitaire, notamment). }\end{array}$ \\
\hline $\begin{array}{l}\text { Continuité péda- } \\
\text { gogique }\end{array}$ & --- & $X$ & --- & $X$ & $\begin{array}{l}\text { Maintien des apprentissages scolaires et des } \\
\text { relations entre l'enseignant et les élèves par la } \\
\text { mise en place de dispositifs et d'outils variés } \\
\text { pour assurer un enseignement momentané- } \\
\text { ment réalisé à distance. }\end{array}$ \\
\hline Corona bond & --- & $X$ & --- & --- & $\begin{array}{l}\text { Obligation émise par l'UE afin de répondre à } \\
\text { la crise économique provoquée par la pandé- } \\
\text { mie de Covid-19. }\end{array}$ \\
\hline
\end{tabular}

4 Nous avons éliminé par exemple le TLFi car sa ressource (TLF) est terminée depuis 1994 et « n’a pas vocation à être mis à jour » et « les définitions qui s'y trouvent ne rendent pas compte des évolutions de la société » (http://atilf.atilf.fr/,[11-08-2021]).

5 L'astérisque veut dire que le mot n'est pas entré dans le dictionnaire à la suite de la pandémie de Covid-19, il y avait déjà figuré avant 2021.

6 L’astérisque veut dire que le mot figure dans le dictionnaire mais avec un sens différent.

7 Il n'y a pas d'article consacré au terme cas contact dans le Petit Larousse illustré, cependant celui-ci apparaît dans l'article consacré à la quatorzaine. 


\begin{tabular}{|c|c|c|c|c|c|}
\hline Coronapiste & --- & $x$ & --- & $x$ & $\begin{array}{l}\text { Piste cyclable provisoire créée afin de favori- } \\
\text { ser la pratique du vélo (adaptée à la distan- } \\
\text { ciation physique) et d'éviter la propagation de } \\
\text { l'épidémie de Covid-19. }\end{array}$ \\
\hline $\begin{array}{l}\text { Couverture vac- } \\
\text { cinale }\end{array}$ & $x$ & $X^{*}$ & $x$ & $x$ & $\begin{array}{l}\text { Proportion de personnes effectivement vacci- } \\
\text { nées dans une population cible au cours d'une } \\
\text { période donnée. }\end{array}$ \\
\hline $\begin{array}{l}\text { Couvre-feu sani- } \\
\text { taire }\end{array}$ & --- & $x$ & --- & --- & $\begin{array}{l}\text { Couvre-feu instauré afin de limiter la pro- } \\
\text { pagation d'une épidémie dans le cadre de } \\
\text { l'état d'urgence sanitaire. }\end{array}$ \\
\hline Crash-test & ---* & $x$ & ---* & ---* & $\begin{array}{l}\text { Tout évènement grave permettant de tester la } \\
\text { résilience et la capacité d'adaptation de qqn., } \\
\text { d'un groupe. }\end{array}$ \\
\hline $\begin{array}{l}\text { Déplacement déro- } \\
\text { gatoire }\end{array}$ & --- & $x$ & --- & --- & $\begin{array}{l}\text { Déplacement contrevenant à une interdiction } \\
\text { générale ou partielle et soumis de ce fait à } \\
\text { justification. }\end{array}$ \\
\hline Dépositoire & $X^{*}$ & $x$ & $x$ & --- & $\begin{array}{l}\text { Local situé hors de l'enceinte du cimetière et } \\
\text { permettant le dépôt des cercueils en attente } \\
\text { d'inhumation ou de crémation. }\end{array}$ \\
\hline Distanciel adj., n. & $x$ & --- & $x$ & $x$ & $\begin{array}{l}\text { Qui a lieu sans présence physique des per- } \\
\text { sonnes concernées. }\end{array}$ \\
\hline $\begin{array}{l}\text { Drive } \\
\text { Recomm. off. } \\
\text { 1. retrait automo- } \\
\text { bile } \\
\text { 2. point de retrait }\end{array}$ & $X^{*}$ & $x$ & --- & $x$ & $\begin{array}{l}\text { 1. Service permettant de retirer une com- } \\
\text { mande tout en restant à bord de son véhicule. } \\
\text { 2. Lieu où s'effectue ce retrait }\end{array}$ \\
\hline Essai randomisé & --- & $x$ & $x$ & --- & $\begin{array}{l}\text { Essai thérapeutique dont les participants sont } \\
\text { répartis aléatoirement dans deux ou plusieurs } \\
\text { groupes qui reçoivent des traitements diffé- } \\
\text { rents. }\end{array}$ \\
\hline $\begin{array}{l}\text { État d'urgence } \\
\text { sanitaire }\end{array}$ & --- & $x$ & $x$ & $x$ & $\begin{array}{l}\text { Situation de catastrophe sanitaire, justifiant, } \\
\text { de par sa gravité, la mise en place par le } \\
\text { gouvernement de mesures exceptionnelles et } \\
\text { temporaires. }\end{array}$ \\
\hline Manuporté & --- & $x$ & --- & $x$ & $\begin{array}{l}\text { Se dit d'une maladie infectieuse, appelée } \\
\text { autrefois maladie des mains sales, dont la } \\
\text { transmission se fait par simple contact. }\end{array}$ \\
\hline Masque & ---* & $x$ & $---*$ & $x$ & $\begin{array}{l}\text { Protection textile couvrant le nez et la bouche, } \\
\text { portée pour limiter la transmission d'une mala- } \\
\text { die infectieuse ou d'inhalation de particules } \\
\text { toxiques. }\end{array}$ \\
\hline Monde d'après & --- & $x$ & --- & $x$ & $\begin{array}{l}\text { Société nouvelle que les gens espèrent bâtir à } \\
\text { la suite de la crise majeure due à la pandémie } \\
\text { de COVID-19. }\end{array}$ \\
\hline Nasopharynx & $x$ & $X^{*}$ & $x$ & $\mathrm{X}^{*}$ & Rhinopharynx. \\
\hline Nébulisation & $---*$ & $x$ & $---*$ & $---*$ & $\begin{array}{l}\text { Pulvérisation très fine d'un produit bactéri- } \\
\text { cide ou virucide dans une pièce, un local, un } \\
\text { véhicule de transport en commun, etc. afin de } \\
\text { les désinfecter. }\end{array}$ \\
\hline
\end{tabular}




\begin{tabular}{|c|c|c|c|c|c|}
\hline Oxymètre & $x$ & $X^{*}$ & $X^{*}$ & $X^{*}$ & $\begin{array}{l}\text { Appareil qui mesure le taux d'oxygène d'un } \\
\text { mélange gazeux ou d'un liquide. }\end{array}$ \\
\hline Paiement mobile & --- & $x$ & $x$ & --- & $\begin{array}{l}\text { Paiement sans contact réalisé au moyen d'un } \\
\text { smartphone, lequel intègre dans sa mémoire } \\
\text { une carte bancaire. }\end{array}$ \\
\hline Pauci- & $x$ & --- & --- & $x$ & $\begin{array}{l}\text { Élément du latin = un petit nombre de. } \\
\text { Paucisymptomatique. }\end{array}$ \\
\hline Pic épidémique & --- & $x$ & $x$ & --- & $\begin{array}{l}\text { Moment où le nombre de cas d'une maladie } \\
\text { contagieuse atteint son maximum et à partir } \\
\text { duquel l'épidémie décroît. }\end{array}$ \\
\hline Plage dynamique & --- & $x$ & --- & --- & $\begin{array}{l}\text { Plage réservée uniquement à la baignade, au } \\
\text { sport et à la promenade (interdiction de s'as- } \\
\text { seoir ou s'allonger pour favoriser le respect } \\
\text { des mesures sanitaires). }\end{array}$ \\
\hline $\begin{array}{l}\text { Plateau épidé- } \\
\text { mique }\end{array}$ & --- & $x$ & $x$ & --- & $\begin{array}{l}\text { Phase d'une épidémie où le nombre de cas } \\
\text { n'augmente plus, où le nombre de personnes } \\
\text { contaminées par jour stagne. }\end{array}$ \\
\hline $\begin{array}{l}\text { Point de bascule } \\
\text { /de basculement }\end{array}$ & --- & $x$ & $x$ & $X^{*}$ & $\begin{array}{l}\text { Moment où un phénomène d'abord rare se } \\
\text { généralise, où un changement minime fait } \\
\text { basculer un système dans un état entièrement } \\
\text { nouveau (= seuil critique). }\end{array}$ \\
\hline Porteur sain & $X^{*}$ & $x$ & $X^{*}$ & $x$ & $\begin{array}{l}\text { Personne contaminée par un agent infectieux } \\
\text { qui ne présente pas de symptômes de mala- } \\
\text { die mais risque de la transmettre à d'autres } \\
\text { personnes. }\end{array}$ \\
\hline $\begin{array}{l}\text { Prélèvement naso- } \\
\text { pharyngé }\end{array}$ & --- & $x$ & --- & $x$ & $\begin{array}{l}\text { Prélèvement de cellules et de micro- } \\
\text {-organismes à l'aide d'un écouvillon introduit } \\
\text { dans le nez afin d'effectuer un test diagnos- } \\
\text { tique (PCR, par ex.). }\end{array}$ \\
\hline Quatorzaine & --- & $x$ & ----* & $x$ & $\begin{array}{l}\text { Isolement de quatorze jours que doivent res- } \\
\text { pecter une personne atteinte de Covid-19 et } \\
\text { les personnes ayant été en contact avec elle. }\end{array}$ \\
\hline Réa & $X^{*}$ & $x$ & $---*$ & $x$ & Fam. Réanimation. \\
\hline Reconfinement & $X^{*}$ & $x$ & --- & $x$ & $\begin{array}{l}\text { Nouveau confinement sanitaire; période } \\
\text { pendant laquelle il s'applique. }\end{array}$ \\
\hline SARS-CoV-2 & --- & $x$ & $x$ & $x$ & $\begin{array}{l}\text { Coronavirus responsable d'une maladie infec- } \\
\text { tieuse très contagieuse (Covid-19), à l'origine } \\
\text { d'une pandémie mondiale. }\end{array}$ \\
\hline Saturomètre & $x$ & --- & $x$ & $x$ & $\begin{array}{l}\text { Appareil qui mesure la saturation du sang en } \\
\text { oxygène. }\end{array}$ \\
\hline Taux de létalité & $X^{*}$ & $x$ & $X^{*}$ & $X^{*}$ & $\begin{array}{l}\text { Rapport du nombre des décès au nombre des } \\
\text { personnes atteintes par une maladie donnée. }\end{array}$ \\
\hline Télétravailler & $X^{*}$ & $x$ & $x$ & $x$ & $\begin{array}{l}\text { Exercer une activité professionnelle à distance } \\
\text { (d'une entreprise), grâce aux outils de télé- } \\
\text { communication. }\end{array}$ \\
\hline Test sérologique & --- & $x$ & $x$ & --- & $\begin{array}{l}\text { Test biologique effectué sur le sérum sanguin } \\
\text { visant à détecter et mesurer les anticorps } \\
\text { spécifiquement dirigés contre une maladie } \\
\text { infectieuse ou parasitaire. }\end{array}$ \\
\hline
\end{tabular}




\begin{tabular}{|l|l|l|l|l|l|}
\hline Vaccinodrome & X & --- & --- & X & $\begin{array}{l}\text { Centre de vaccination collective aménagé } \\
\text { dans un vaste espace. }\end{array}$ \\
\hline Ventilateur & X & $---*$ & X & ---* & Respirateur. \\
\hline VPN & X & X & X & X & $\begin{array}{l}\text { Sigle anglais de Virtual Private Network } \\
\text { (réseau privé virtuel). Système qui permet une } \\
\text { activité en ligne privée et sécurisée, en isolant } \\
\text { et en cryptant les données. }\end{array}$ \\
\hline
\end{tabular}

Tableau 2 : Les autres termes entrés dans le millésime 2022 du Petit Robert ou du Petit Larousse illustré et figurant aussi dans les dictionnaires en ligne choisis

Cette deuxième liste prouve aussi que la crise sanitaire est omniprésente dans les nouveaux mots et sens faisant leur entrée dans les dictionnaires Le Petit Robert et Le Petit Larousse illustré et que le Grand dictionnaire terminologique et le Wiktionnaire sont aussi réactifs à la naissance de ce vocabulaire. En grande majorité, ces nouveaux mots ou sens sont d'origine médicale ou sont le reflet de la pandémie dans la vie quotidienne et touchent ainsi le travail ou les activités des gens. Il y a deux ans, presque personne n'utilisait les termes comme nasopharynx, porteur sain, télétravailler, VPN et d'autres, pourtant, aujourd'hui, ils figurent dans tous les dictionnaires consultés. Dans la plupart des cas, ils y font leur apparition sous un nouveau sens. Ainsi les mots dépositoire, drive, nébulisation, etc., qui figuraient déjà dans les dictionnaires, voient leurs nouveaux sens précisés.

À première vue, il peut sembler que le Petit Larousse illustré et le Wiktionnaire sont les plus réactifs et intègrent le plus grand nombre de mots et sens nouveaux liés à la pandémie. Néanmoins, nous sommes arrivée à la conclusion que les résultats obtenus ne sont pas comparables et qu'il n'est pas possible de donner des chiffres précis. Par exemple, le terme couvre-feu figure dans tous les dictionnaires consultés (avec la définition correspondant partiellement à celle du couvre-feu sanitaire mentionnée dans le tableau 2) mais parce qu'il a fait son apparition dans le Petit Larousse illustré dans la locution adjectivale couvre-feu sanitaire, nous avons noté son absence dans les trois dictionnaires restants.

Il ressort aussi des deux tableaux que c'est la locution qui est courante pour donner un autre sens à un mot déjà existant, souvent pour préciser et transformer le réel. Ainsi la distanciation s'emploie avec les adjectifs physique ou sociale, l'immunité devient collective, l'état d'urgence est sanitaire, la plage peut être ouverte en mode dynamique, le prélèvement peut être aussi nasopharyngé et le déplacement contrevenant à une interdiction est dérogatoire. Il en va de même pour les noms accolés d'un complément du nom, par exemple cas contact pour décrire les potentialités de propagation du virus entre les individus ou le monde d'après dont l'idée a émergé aussi bien dans les discours politiques que dans la presse.

Il faut également constater qu'il y a une spécificité française de la création lexicale. Même si la pandémie de Covid-19 est d'envergure internationale et beaucoup de termes liés à celle-ci sont empruntés à l'anglais, il semble que les Français tentent même pendant cette période difficile de résister à la pression des anglicismes. Ainsi, le traçage remplace le tracking dans les dictionnaires français, le terme click and collect fait son apparition dans le Petit Larousse illustré ainsi que dans le Grand dictionnaire terminologique et le Wiktionnaire, mais celui-ci n'éclipse pas 
le cliqué-retiré, sa version française officiellement recommandée, qui a aussi fait son entrée dans le Petit Larousse illustré. De même, le foyer de contagion supplante l'anglicisme cluster.

\section{Conclusion}

La crise sanitaire qui touche le monde entier, y compris la France, s'accompagne d'un vocabulaire spécial. Certains des termes existaient déjà dans les dictionnaires mais ont pris une autre signification. D’autres, souvent empruntés à l'anglais, sont nouveaux. Peu ou pas utilisés auparavant, au cours de la pandémie, ils se sont généralisés dans le vocabulaire des Français. Ces derniers (de même que tous les autres peuples) sont devenus des spécialistes en matière de maladie à coronavirus. Aujourd'hui, en employant par exemple le terme masque, personne ne pense plus au carnaval ou à la comédie culte The Mask (Le Masque) avec Jim Carrey dans le rôle principal. Tout le monde parle de masques de protection, d'hygiène et de respiration et même des termes comme FPP2 ou FPP3 sont couramment utilisés. Il n'est donc pas étonnant que les nouveaux entrants au Petit Robert et au Petit Larousse illustré reflètent cette nouvelle réalité et ce nouveau vocabulaire covidien que l'on doit non seulement à la sphère médicale ou politique mais aussi à la créativité des journalistes et qui est rendu vivant par les usagers.

Dans de nombreux cas, il s'agit des termes dont l'usage était auparavant réservé à d'autres domaines qu'au milieu médical. Plusieurs d'entre eux proviennent par exemple du langage militaire. Ainsi, l'adjectif aéroporté s'associait jusqu'à la crise seulement aux troupes de l'armée, désormais, on parle aussi de virus aéroporté. Beaucoup de mots déjà existants ont été repensés. À titre d'exemple, nous pouvons mentionner la quarantaine, indiquant une mise à l'isolement de personnes pour empêcher la propagation d'une maladie, qui est devenue quatorzaine car le terme quarantaine ne correspondait pas à la réalité du Covid-19 pour lequel l'incubation est de 14 jours (sa durée a été ramenée plus tard à 7 jours, dans ce cas, on parle de septaine). Plusieurs nouveaux termes ont été formés par dérivation ou composition. Ainsi, le confinement s'est changé par l'ajout du préfixe dé- (exprimant la cessation) en déconfinement, puis en reconfinement pour indiquer, en ajoutant le préfixe re-, un nouveau confinement sanitaire. Ce sont aussi plusieurs fractolexèmes qui témoignent de la productivité actuelle de fractocomposition : téléa donné naissance à la téléconsultation qui est devenue la pratique courante des médecins et le télétravail qui figurait déjà dans les dictionnaires a été décliné en télétravailler ; corona- voulant dire couronne (pour indiquer la forme arrondie du virus) fait partie du fractocomposé coronapiste, piste cyclable créée au début de la pandémie pour éviter la foule dans les transports en commun, tandis que dans coronapéro, coronamis, coronabdos, etc. (mots n'étant pas encore intégrés par le Petit Robert ou le Petit Larousse illustré) il fait partie des mots-valises.

Notre analyse montre aussi que le nom du virus et celui de la maladie sont un sujet de questionnements. Nous avons constaté une hésitation dans le genre attribué au terme Covid-19, probablement à cause de la confusion entre la dénomination du virus et celle de la maladie. Pour le Petit Robert estimant que «c'est l'usage qui fait loi », Covid-19 est «masculin ou féminin », alors que pour le Petit Larousse illustré, il est « féminin ou masculin », plus correct en féminin, mais masculin chez de nombreux locuteurs. Les deux dictionnaires ne se sont pas non plus mis 
d'accord sur l'usage de la minuscule ou de la majuscule : le Petit Robert privilégie la minuscule, tandis que le Petit Larousse illustré préconise la majuscule.

Les chances de voir apparaître des mots nouveaux dans la nomenclature des dictionnaires dépendent généralement de leur vitalité à long terme. Néanmoins, notre analyse montre que certains termes, notamment ceux créés et diffusés au cours d'un grand événement historique tel que pandémie de Covid-19, peuvent entrer dans les dictionnaires papier ou en ligne en un temps record après leur naissance. Mais tout ce vocabulaire covidien est-il temporaire ou restera-t-il longtemps en usage ? La réponse à cette question ou à d'autres sera sans aucun doute cruciale pour les lexicologues en charge des prochaines éditions des dictionnaires Le Petit Robert et Le Petit Larousse illustré ainsi que pour ceux qui seront en charge de la mise à jour des dictionnaires en ligne.

\section{Bibliographie}

У Boulanger, Jean-Claude, et al. (2003). « Le Petit Robert par lui-même : de l'ombre à la lumière ». In : Jean-Claude Boulanger; Aline Francoeur; Monique C. Cornier (éds.). Les dictionnaires Le Robert. Genèse et évolution. Montréal : Presse de l’Université de Montréal, pp. 157-188.

У BOUZIDI, Boubakeur (2010). « Néologicité et temporalité dans le processus néologique ». Synergies Algérie, 9, pp. 27-36.

У CABRÉ Maria Teresa (1998). La terminologie. Théorie, méthode et applications. Ottawa : Les Presses de l'Université d'Ottawa, Paris : Armand Colin.

\ CUSIN-BERCHE, Fabienne (1999). «La notion d'« unité lexicale » en linguistique et son usage en lexicologie ». Linx. http://journals.openedition.org/linx/730 [05-08-2021].

У ELCHACAR, Mireille; MARTINEZ, Camille (2008). « Une banque de données en ligne pour suivre l'évolution des nomenclatures du Petit Robert et du Petit Larousse, et leur ouverture aux mots du Québec ». Communication, lettres et sciences du langage, 2.1, pp. 79-91.

\ GUILBERT, Louis (1975). La créativité lexicale. Paris : Larousse.

У MORTUREUX, Marie-Françoise (1997). La lexicologie entre langue et discours. Paris : SEDES.

У REY-DEBOVE, Josette (1971). Étude linguistique et sémiotique des dictionnaires français contemporains. Paris : Mouton.

У SABLAYRolles, Jean-François (2000). La néologie en français contemporain. Paris : Champion.

У SAblayrolles Jean-François (2011). «Alain Rey, le linguiste et les mots nouveaux ». In : François GAUDIN (éd.). Alain Rey, vocabuliste français. Limoges : Éditions Lambert-Lucas, pp. 87-98.

У SABLAyrolles, Jean-François (2017). Les néologismes : créer des mots français au-jourd'hui. Paris : Éditions Garnier, en partenariat avec Le Monde.

У SAblayrolles, Jean-François (2019). Comprendre la néologie. Conceptions, analyses, emplois. Limoges : Lambert-Lucas.

\section{Sitographie}

У Académie française. Le covid 19 ou La covid 19. https://www.academie-francaise.fr/le-covid-19-ou-la-covid-19 [11-08-2021].

ע Club d'orthographe de Grenoble. Mots nouveaux des dictionnaires. https://orthogrenoble.net/mots-nouveaux-dictionnaires_[27-08-2021]. 
y Larousse. https://www.fortissimots.com/wp-content/uploads/DP-motsnouveaux2022-Larousse.pdf [14-08-2021].

$\searrow$ Le Grand dictionnaire terminologique. http://gdt.oqlf.gouv.qc.ca/ [25-08-2021].

\ Le Robert, dico en ligne. Covid. https://dictionnaire.lerobert.com/definition/covid [12-08-2021].

$\checkmark \quad$ Le Robert. Le Robert présente ses dictionnaires et mots nouveaux 2022. https://manuscritdepot.com/dictionnaire_le_robert_mots_nouveaux_2022.pdf [12-08-2021].

y Néoveille. https://tal.lipn.univ-parisl3.fr/neoveille/html/login.php?action=login\# [30-08-2021].

> Wiktionnaire. https://fr.wiktionary.org/wiki/Wiktionnaire:Page_d'accueil [15-08-2021].

\section{Dagmar Koláříková}

Katedra románských jazyků

Fakulta filozofická

Západočeská univerzita v Plzni

Riegrova 11

30614 PLZEŇ

République tchèque 